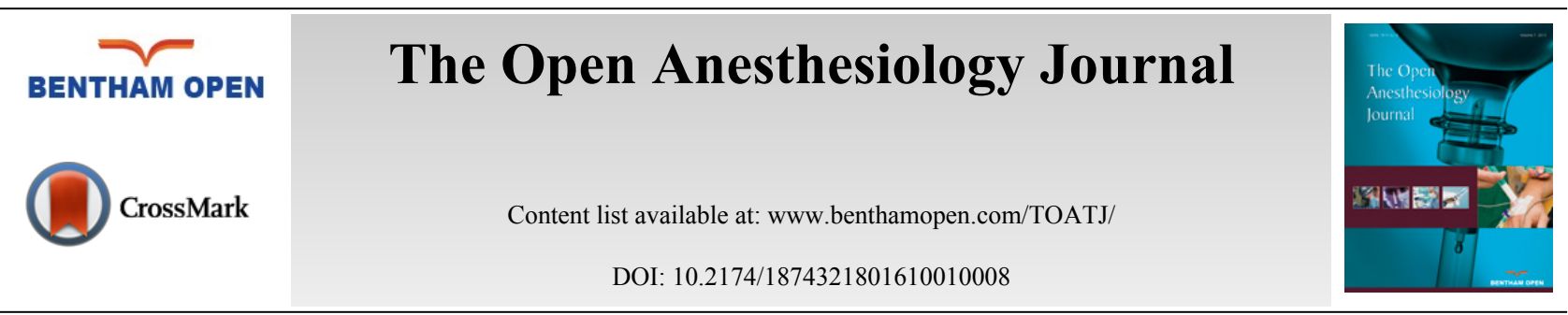

\title{
Technical Challenges of Spinal Cord Stimulation Lead Placement in Morbidly Obese Patients
}

\author{
Sanjay Sastry ${ }^{1, *}$, David Nussear ${ }^{2}$, Albert Gillespy $^{3}$ and Kristina Berger ${ }^{4}$ \\ ${ }^{I}$ Pain Management, Coastal Neurology \& Pain Centers, 801 Beville Road, South Daytona, Florida 32119, United \\ States \\ ${ }^{2}$ Anesthesiology Lake Mary Surgery Center 460 St. Charles Court, Lake Mary, Florida 32748, United States \\ ${ }^{3}$ Orthopedic Clinic of Daytona Beach, 1890 LPGA Boulevard, Suite 24, Daytona Beach, Florida 32117, United States \\ ${ }^{4}$ Lake Mary Family Practice, 755 Rinehart Rd. \#100, Lake Mary, Florida 32746, United States
}

Received: November 12, 2014

Revised: February 7, 2016

Accepted: February 18, 2016

\section{Abstract: \\ Background and Aims:}

This paper details the technical challenges involved in the placement of spinal cord stimulator (SCS) leads in morbidly obese patients. It also provides tips for aiding in placement of these devices.

\section{Objective:}

This paper aims to suggest techniques which may assist in solving commonly occurring difficulties in SCS placement in the morbidly obese patient.

Design:

Technical note

Setting:

These challenges commonly occur in an outpatient surgery setting or hospital setting.

\section{Conclusion:}

There are an increasing number of morbidly obese patients that require a SCS for chronic pain control management. Techniques have been identified to overcome these challenges in placement of SCS leads.

Keywords: Body Mass Index (BMI), Chronic pain, Morbid obesity, Spinal Cord Stimulation (SCS).

\section{INTRODUCTION}

Increasing rates of obesity in both Western and developing countries result in rising patient populations referred for chronic pain management. The use of spinal cord stimulation offers a long term solution for management of these patients. However, these patients present a unique set of both physical and technical challenges to the clinician. This paper will present guidelines and insights as to how to best manage these patients during lead placement of spinal cord stimulators (SCS).

Nearly two out of every three adults in the United States is overweight [1].

\footnotetext{
* Address correspondence to this author at the Pain Management Coastal Neurology \& Pain Centers, 801 Beville Road, South Daytona, Florida 32119, United States; E-mail: sjsastry@hotmail.com
} 
Experts often rely on a formula called the body mass index (BMI) to determine if a person is overweight. The BMI estimates your level of body fat based on your height and weight.

- A BMI from 18.5 to 24.9 is considered normal.

- Adults with a BMI of 25 to 29.9 are considered overweight. Since the BMI is an estimate, it is not accurate for all people. Some people in this group, such as athletes, may have a lot of muscle weight, and therefore not as much fat. These people will not have an increased risk of health problems due to their weight [2].

- Adults with a BMI of 30 to 39.9 are considered obese.

- Anyone with a BMI greater than or equal to 40 is considered extremely obese.

- Anyone more than 100 pounds overweight is considered morbidly obese.

Both men and women with BMI of $30 \mathrm{~kg} / \mathrm{m} 2$ or higher were twice as likely to have difficulties in performing a range of basic daily physical activities. Compared to women with BMI of lower than $25 \mathrm{~kg} / \mathrm{m} 2$, those with BMI of 30 $\mathrm{kg} / \mathrm{m} 2$ or higher were 1.5 times more likely to have symptoms of intervertebral disk herniation [3].

\section{PHYSICAL CHALLENGES TO SPINAL CORD STIMULATOR LEAD PLACEMENT}

Firstly, can the patient lie prone for one to two hours? Due to the obese abdomen, the tidal volume and functional residual capacity (FRC) are reduced in the prone position [4]. There is also aortocaval compression, which might decrease the overall cardiac output [5]. Breathing may further decrease secondary to sinus congestion [6]. A great majority of such patients have not attempted to lay prone in years. Once it is determined that the patient is going to proceed with a spinal cord stimulation procedure, encourage them to try to lay prone and supine daily to increase the duration prior to the surgery date. Encourage the patient to lay prone daily for 15 minutes and to increase in increments the time by 15 minutes daily.

Second is the raw weight of the patient. Weight of the patient cannot exceed the maximum allowable limit of the procedure table. The use of specialty bariatric operating tables may be required, as long the use of fluoroscopic imaging is not precluded [7].

A third physical challenge in placement of the spinal cord stimulator leads in the morbidly obese patients has to do with the patients' skin. Multiple and thorough applications of antiseptic solutions on the skin in between the folds is necessary to reduce incidence of infection. Instruct the patient to have a thorough shower or bath using soap and hot water to wash all the skin folds the evening before and the morning of the procedure. Perioperative and postoperative use of antibiotics may reduce incidence of infection.

\section{TECHNICAL CHALLENGES TO SPINAL CORD STIMULATOR LEAD PLACEMENT}

Generally, there are two stages to spinal cord stimulator placement, the trial and the permanent. In the United States, the majority of the trials or temporary stimulators are performed using percutaneous approach to place cylindrical leads. It is important to obtain a flat plane, prone position for both of these stages. This is necessary in order to obtain clear fluoroscopic images for the visualization of anatomical landmarks, as well as to facilitate entry of the needle into the epidural space. Due to an increase of subcutaneous fatty tissue, positioning can be a challenge in the obese patient. This is related to the physiological lordosis from the pannus forcing the back into concavity. Positioning devices, including several pillows, may prove to be helpful in achieving a more neutral position. Many times it is necessary to adjust the patients' panniculus to accommodate a neutral, anterior posterior position,

The positioning challenges, coupled with the tissue depth necessary to access the epidural space, create the most challenging technical scenario with these patients. A 4-inch epidural needle is typically used to access the epidural space in normal sized people The obese patient will almost always necessitate the use of a 6-inch epidural needle. This will provide for a flatter needle plane as to produce 30 degrees or less of an angle. This will allow adequate access into the epidural space. The skin entry point might be required to be lower than with the use of a 4-inch epidural needle. For example, the T12-L1 epidural space may require access starting from either side of the L4 pedicle. This might produce a more optimum angle of entry for the needle trajectory into the epidural space; a longer needle would be necessary to reach that distance.

Once the lead or leads are placed in the ideal respective sites, prevention of lead migration will be the next challenge. This concern needs to be a priority, especially with the morbidly obese. After the epidural needle is 
withdrawn, suturing the leads to the skin using a 2-O nylon suture might reduce the chance of lead movement as compared to the use of tape or adhesives alone. Using adhesives such as tincture of benzoin, or surgical tape such as bio-occlusive film or steri-strips might be ineffective if used alone. This is due to the fact that the patients with loose skin will break down tape and moisture may accumulate from the taped area on the back. This will further reduce the adhesive nature between tape to the lead, increasing the incidence of lead migration. The trial period in such patients should be as short (less than 72 hours) as possible in order to reduce chances of lead migration not providing adequate paresthesia for pain relief and as a possible site for infection.

Once the patient has successfully completed the trial spinal cord stimulator, further challenges are noted for permanent implantation of the stimulator. Ten centimeters is the average length for a cut down required for lead placement. In a morbidly obese patient, the length of the incision for a cut down might be extended to 12 centimeters. Awareness of hemostasis is paramount when dissecting the subcutaneous layer of fat in order to gain access to the fascia. In order to acquire a proper 15 to 20 degree angle for the needle placement, an extended incision may allow the ability to lay the needle more flat. Also, when an incision is extended in the morbidly obese patient, the ability to view the fascial plane is enhanced. Once the leads are in the desired location of the epidural space, anchoring the leads to the fascia is also easier with an extended incision with adequate retraction.

Proper retraction of the sides of the incision due to the increased depth of the wound is necessary; otherwise, the subcutaneous fat layer will hinder proper dissection and lead anchoring. Depending on the anchor chosen, a longer suture driver may be necessary to properly guide the suture to the fascia and to subsequently suture the fascia to the lead as an anchor.

Once anchoring of the leads is done, another incision is required for implantation of the generator. Due to the ample subcutaneous fat available, it is easy to get lost and dissect too deep. It is important to remind oneself to dissect the pocket approximately $2 \mathrm{~cm}$ in depth. Once the tunneling is performed and leads are attached to the generator, prior to closing the wounds irrigation, preferably using antibiotic solution, is recommended. This is in order to minimize the incidence of postoperative wound infections. If the pocket of the generator is too large, there is a higher chance that the generator could flip over once the patient starts mobilizing. Also, if the generator is placed too deep, recharging and programming is more difficult. A pocket that is "snug' and $2 \mathrm{~cm}$ from the skin is always preferred.

In the surgical technique of performing a laminectomy for the implantation of paddle leads of a permanent spinal cord stimulator, appropriate anesthesia technique is a challenge. If general anesthesia is selected, there may be elevated peak pressures which may lead to a pneumothorax. Physiologic circulation of the cardiovascular system will be impaired under general anesthesia, and cardiac output may decrease. Increase in overall physical injuries may also occur in positioning of the patient from supine to prone position. Local anesthesia with IV sedation (Monitored Anesthesia Care $=$ MAC) may be a better option. Physiologic changes are not as drastic when a patient is sedated and spontaneously breathing. The laminectomy is easier to perform with a slightly larger incision.

Once the wounds are closed, infections are a major cause of concern in the morbidly obese patient. A postoperative course of antibiotics covering cutaneous bacterial organisms will decrease the likelihood of infections. Also, special instructions should be given to such patients about keeping the wounds dry and maintaining proper hygiene.

Eventually, once the patient is feeling better and receiving analgesia from the spinal cord stimulator, an exercise program to encourage weight loss should be a priority.

It is also important to note that prior to any surgical intervention informed consent must be obtained from the patient. A detailed review of the higher risks associated with obesity is essential to the consent process.

\section{CONCLUSION}

A total of forty-two (42) morbidly obese patients were implanted with a spinal cord stimulator using the techniques aforementioned. One patient had wound dehiscence that eventually closed by secondary intervention. One other patient had lead migration requiring revision of SCS placement. The patients had good pain relief from SCS therapy resulting in successful analgesia.

The morbidly obese patient presenting for both trial and permanent SCS offers the clinician a very unique set of challenges. As presented in this paper, these challenges can be overcome, offering these patients long term effective pain relief. 


\section{CONFLICT OF INTEREST}

The authors confirm that this article content has no conflict of interest.

\section{ACKNOWLEDGEMENTS}

Declared none.

\section{REFERENCES}

[1] Flegal KM, Carroll MD, Kit BK, Ogden CL. Prevalence of obesity and trends in the distribution of body mass index among US adults, 1999-2010. J Am Med Assoc 2012; 307(5): 491-7. [PMID: 22253363]

[2] Ogden CL, Carroll MD, Kit BK, Flegal KM. Prevalence of obesity and trends in the distribution of body mass index among US children and adolescents, 1999-2010. J Am Med Assoc 2012; 307(5): 483-90. [PMID: 22253364]

[3] Lean ME, Han TS, Seidell JC. Impairment of health and quality of life using new US federal guidelines for the identification of obesity. Arch Intern Med 1999; 159(8): 837-43.

[PMID: 10219929]

[4] Pelosi P, Croci M, Ragavan I, et al. The effects of body mass on lung volumes, respiratory mechanics, and gas exchange during general anesthesia. Anesth Anal 1998; 64: 181-7. [PMID: 9728848 ]

[5] Blass NH. Regional anesthesia in the morbidly obese. Reg Anesth 1979; 4(3): 20-02.

[6] Riggs A. Congestion Conundrum. USA: Massage and Bodywork 2012; pp. 29-30.

[7] Uppot RN, Sahani DV, Hahn PF, Gervais D, Mueller PR. Impact of obesity on medical imaging and image-guided intervention. AJR Am J Roentgenol 2007; 188(2): 433-40. [PMID: 17242253]

(C) Sastry et al.; Licensee Bentham Open.

This is an open access article licensed under the terms of the Creative Commons Attribution-Non-Commercial 4.0 International Public License (CC BY-NC 4.0) (https://creativecommons.org/licenses/by-nc/4.0/legalcode), which permits unrestricted, non-commercial use, distribution and reproduction in any medium, provided the work is properly cited. 